\title{
124. Properties of Excitable Membrane Produced on the Surface of Protoplasmic Drop in Nitella
}

\author{
By Isao Inoue,**) Yoshio Ishima,*) Hidenori HorIE,*) \\ and Toshifumi TAKenAKA*)
}

(Comm. by Masahiro OkAdA, M. J. A., June 12, 1971)

The properties of the protoplasmic drop which was produced by amputating Nitella internode in the artificial cell sap solution have been investigated by Kamiya et $a ._{.}{ }^{1)-3), 8)}$ According to their studies, thin precipitated membrane was formed on the surface of the protoplasmic drop and the property of this membrane was semipermeable to inorganic ions. This membrane would be assumed to be made of the substance in the protoplasm and directly contact with outside medium. Therefore, it will be a favourable material for studying the excitability of the membrane. The purpose of this paper is to study the properties of this excitable membrane by using the method of electrophysiology, electron microscopy and biochemistry.
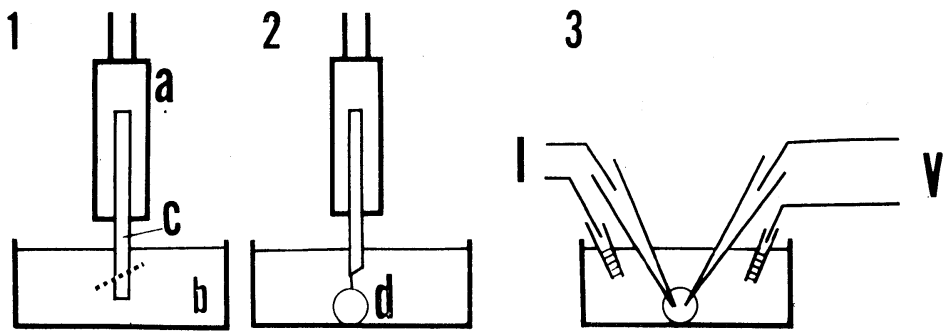

Fig. 1. Schematic illustration of experimental procedures for making a protoplasmic drop and recording the electrical responses of the drop membrane.

1: Setup for amputating Nitella internode. a: Lucite holder filled with solution, b: Lucite vessel filled with solution, $c$ : Internodal cell of Nitella. Broken line indicates the locus where amputation is to be conducted. 2: Effusion of protoplasm in the form of a continuous thread from the lower opening of the internodal cell. d: Protoplasmic drop. 3: Setup for measuring electrical responses. Two microelectrodes are inserted into the protoplasmic drop, one for supplying current (I) and the other for recording membrane potential (V).

*) Department of Physiology, Tokyo Medical and Dentaly University, Yushima, Bunkyo-ku, Tokyo.

**) Department of Pharmaceutical Science, Hokkaido University, Sapporo. 
Internodal cells of Nitella flexilis and Nitellopsis obtusa which were collected at Lake Yunoko, Tochigi-Ken and Lake Kawaguchiko, Yamanashi-ken, were used throughout this experiment. The experimental procedure is schematically shown in Fig. 1. An internodal cell was held vertically in a holder $a$ and a small portion of its lower end submerged in the solution of vessel $b$ (Fig. 1-1). The medium solution of holder $a$ and vessel $b$ was $1.0 \mathrm{mM} \mathrm{Ca}\left(\mathrm{NO}_{3}\right)_{2}, 0.5 \mathrm{mM}$ $\mathrm{MgSO}_{4}, 0.1 \mathrm{mM} \mathrm{KNO}$ and a small amount of Na-phosphate buffer. The $\mathrm{pH}$ of this solution was 6.8-7.2. The tonicity of this medium which was adjusted with mannitol solution was slightly hypotonic to the cell sap solution in order to prevent plasmolysis. When the cell was amputated in a solution of vessel $b$, the protoplasm flowed down from the opening of the cell and formed a protoplasmic drop on the bottom of the vessel (Fig. 1-2). The internodal cell was removed from the solution in the vessel when the diameter of the protoplasmic drop reached approximately $300 \mu$ by a continuous accumulation of protoplasm. The drop of $300 \mu$ in diameter was a convenient size for measuring electrical responses. The solution of the vessel was changed at least three times with the same solution in order to eliminate the cell sap solution. For measuring electrical responses two microelectrodes were inserted into the drop (Fig. 1-3), one for supplying current and the other for recording the membrane potential. The reference electrode was silver-silver chloride- $\mathrm{KCl}$ agar electrodes placed in the solution of the external medium. Experiments were carried out at $18-22^{\circ} \mathrm{C}$.

Electron microscopic study of the protoplasmic drop. The protoplasmic drop was fixed in formalin for over night and immersed in $1 \%$ osmium tetroxide for a day. After dehydration through an ethanol series the specimens were embeded in epoxy resin. The sections were cut on a Poter-Blum microtome, and stained with uranyl and lead. Observations were made with Hitachi HS7 electron microscope. An electron micrograph of the protoplasmic drop is shown in Fig. 2. A continuous electron dense line in which one would recognize periodic globular structure and partial unit membrane structures was observed on the surface of the drop. This electron dense line makes a continuous closed circuit around the drop under the observation of low magnification. Furthermore, no connective tissue was observed outside of the surface membrane.

Phospholipids and amino acids analysis of the protoplasm. The phospholipids of the protoplasm obtained from Nitellopsis obtusa was analyzed by using the method of two-dimensional thin-layer chromatography which was conducted on silica gel.5) Approximately 


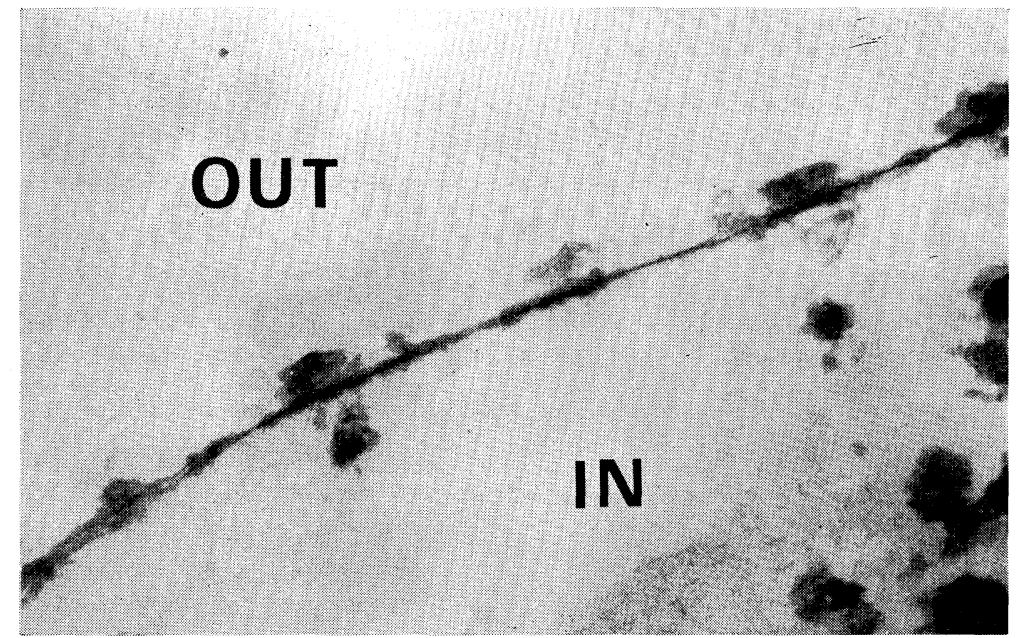

Fig. 2. Electron micrograph of protoplasmic drop of Nitellopsis obtusa. $\times 80000$

$0.5 \mathrm{~g}$ of the wet protoplasm was homogenized in 20 volumes of chloroform-methanol $2: 1$. The solvent was removed from the extract on a rotating evaporator under reduced pressure at $35^{\circ} \mathrm{C}$ and the total lipid sample was taken up in a small volume of chloroform-methanol $2: 1$. The plate, on which the lipid extract was applied as a spot, was developed in chamber lined with solvent-saturated filter paper. For the solvents we used chloroform-methanol-water-28\% aqueous ammonia $130: 70: 8: 0.5$ in the first dimension followed by chloroform-

Table I. Amino acid composition of protoplasm in Nitellopsis obtusa

\begin{tabular}{lc}
\hline & (residues $/ \mathbf{1 0 0 0}$ total amino acid residues) \\
Lys & 70.3 \\
His & 19.5 \\
Arg & 48.5 \\
Asp & 89.6 \\
Thr & 49.7 \\
Ser & 58.9 \\
Glu & 108.2 \\
Pro & 60.2 \\
Gly & 88.6 \\
Ala & 99.8 \\
Cys/2 & 20.7 \\
Val & 76.5 \\
Met & 17.8 \\
Ile & 41.8 \\
Leu & 92.2 \\
Tyr & 24.0 \\
Phe & 33.7 \\
\hline
\end{tabular}


aceton-methanol-acetic acid-water $100: 40: 20: 20: 10$ in the second. After separation of lipids its composition was determined by spraying with reagents. The composition of phospholipids was phosphatidyl choline $(47.7 \%)$, phosphatidyl ethanolamine $(17.6 \%)$, sphingomyelin $(6.8 \%)$, phosphoinositide $(5.9 \%)$, phosphatidic acid $(3.3 \%)$ and $\mathrm{X}$ (proteolipid; 18.7\%). Phosphatidyl serine could not be detected in this method. Amino acid analyses of the protoplasm were performed on a commercial model (JLC-5AH, Hitachi) of the automatic amino acid analyser. In these procedures we followed the method of Stein and Moore. ${ }^{4)}{ }^{6)}$ Amino acid composition is presented in Table I.

Electrical responses. Various type of electrical responses was recorded from the protoplasmic drop. Typical three types of electrical responses are shown in Fig. 3. All-or-none action potential hav-
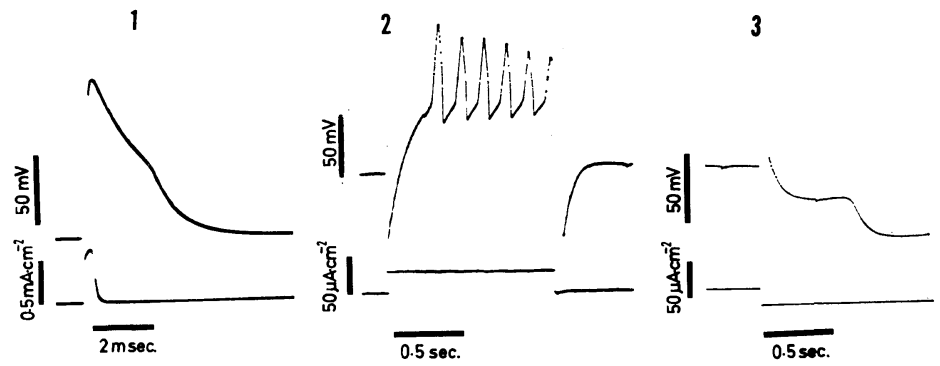

Fig. 3. Electrical responses recorded from the drop membrane. 1: All-or-none action potential, 2: Repetitive firing of action potential by applying long outward current, 3: Hyperpolarizing response. Upper record shows membrane potential and lower record shows applying current in each case.

ing $115 \mathrm{mV}$ in amplitude and $5 \mathrm{msec}$ in duration is shown in Fig. 3-1. The shape of the action potential is similar to that of nerve rather than Nitella internode. Repetitive firing of action potential and hyperpolarizing responses by applying outward and inward current pulses are shown in Figs. 3-2 and 3-3 respectively. However, no clear response was observed immediately after forming the drop. It required more than half an hour until the response appeared. In the early stage after formation of the drop, the resting potential and large value of the membrane resistance were usually not observed. However, the resting potential of $20-50 \mathrm{mV}$ appeared at about one hour after formation of the drop. The membrane resistance and capacity at this stage were $400-900 \Omega \mathrm{cm}^{2}$ and $1 \mu \mathrm{F} \mathrm{cm}^{-2}$.

A continuous electron dense line which was observed on the surface of the protoplasmic drop is assumed to be a membrane. And this membrane would be produced by proteins and phospholipids of the protoplasm. Three types of responses were obtained from the 
membrane by applying electrical stimulation.

Acknowledgement. We wish to express our deep gratitude to Dr. K. Waku for analyzing phospholipids, Prof. Y. Nagai for analyzing amino acids of protoplasm and Mr. H. Miyamoto for photographing the electron micrograph at Tokyo Medical and Dental University. We also wish to thank to Drs. U. Kishimoto, N. Iwasaki, $\mathrm{K}$. Kuroda for their help to this work. This investigation was supported by a grant from the Ministry of Education (Japan).

\section{References}

1) Kamiya, N., and Kuroda, K. (1957): Proc. Japan Acad., 33, 149.

2) - (1957): Proc. Japan Acad., 33, 403.

3) - (1965): Proc. Fourth International Congr. on Rheology, part 4, John Wiley and Sons, 157.

4) Moore, S., and Stein, W. H. (1948): J. Biol. Chem., 176, 337.

5) Parsons, J. G., and Patton, S. (1967): J. Lipid Research, 8, 696.

6) Stein, W. H., and Moore, S. (1948): J. Biol. Chem., 176, 337.

7) Takenaka, T., Inoue, I., Ishima, Y., and Horie, H. (1971): Proc. IUPS, VIII (in press).

8) Yoneda, M., and Kamiya, N. (1969): Plant and Cell Physiol., 10, 821. 